PUPT-1874

hep-th/9906220

\title{
Tachyon Stabilization in the AdS/CFT Correspondence
}

\author{
Igor R. Klebanov \\ Joseph Henry Laboratories \\ Princeton University \\ Princeton, New Jersey 08544 \\ USA
}

\begin{abstract}
We consider duality between type $0 \mathrm{~B}$ string theory on $A d S_{5} \times S^{5}$ and the planar CFT on $N$ electric D3-branes coincident with $N$ magnetic D3-branes. It has been argued that this theory is stable up to a critical value of the ' $t$ Hooft coupling but is unstable beyond that point. We suggest that from the gauge theory point of view the development of instability is associated with singularity in the dimension of the operator corresponding to the tachyon field via the AdS/CFT map. Such singularities are common in large $N$ theories because summation over planar graphs typically has a finite radius of convergence. Hence we expect transitions between stability and instability for string theories in AdS backgrounds that are dual to certain large $N$ gauge theories: if there are tachyons for large AdS radius then they may be stabilized by reducing the radius below a critical value of order the string scale.
\end{abstract}

June 1999 


\section{Introduction}

It has been proposed [1,2, 3, [4] that certain 4-dimensional non-supersymmetric large $N$ gauge theories are dual to backgrounds of the type 0 string theory [5]. This work was motivated by the recently discovered relations between type IIB strings and superconformal gauge theories on $N$ coincident D3-branes [6, 7,8,9,10] as well as by Polyakov's suggestion [11] (building on his earlier work [12]) that the type 0 string theory is a natural setting for extending this duality to non-supersymmetric gauge theories. Since then a number of further studies of D-branes in type 0 theories [13, 14, 15] and the associated dualities [16, 17, 18, 19,20,21,22,23] have appeared.

It is well known that taking the low energy limit on a stack of $N$ D3-branes in flat space and comparing it to the corresponding limit of the 3-brane classical solution suggests that the $\mathcal{N}=4$ supersymmetric $S U(N)$ gauge theory is dual to type IIB strings on $A d S_{5} \times S^{5}$ [8,9,10]. Generalizations of this duality to other 4-dimensional gauge theories follow if the stack of D3-branes is placed on a transverse space $Y_{6}$ which is a cone over an Einstein manifold $X_{5}$ [24, 25, 26, 27, 28,29, 30]. Then the the CFT on the D3-branes is dual to type IIB string theory on $A d S_{5} \times X_{5}$. An interesting set of dualities of this sort is found if $Y_{6}$ is an orbifold $R^{6} / \Gamma$ where $\Gamma$ is a discrete subgroup of $S O(6)$ [25,26]. The corresponding theories on the branes are conformal if the orbifold group acts appropriately on the gauge indices [24]. Duality between such CFT's and type IIB backgrounds of the form $A d S_{5} \times S^{5} / \Gamma$ is thus an orbifold of the original duality between the $\mathcal{N}=4$ theory and the $\operatorname{Ad} S_{5} \times S^{5}$ background. Remarkably, a large class of such CFT's is non-supersymmetric in the large $N$ (planar) limit [25,26]. A phenomenological application of non-supersymmetric CFT's was recently proposed in [31] but later criticized in [32].

Alternatively, one may construct gauge theory/string dualities by stacking D3-branes in string models other than type IIB. Type 0 theories are attractive in this regard but they have a well-known and seemingly fatal flaw in that their spectrum contains a tachyon of $m^{2}=-2 / \alpha^{\prime}$ which is not removed by the non-chiral GSO projection $(-1)^{F+\tilde{F}}=1$. In [1] it was suggested, however, that the tachyon may be stabilized in appropriate type 0 backgrounds which are relevant to gauge theory applications.

The type 0 models have a doubled set of RR fields compared to their type II cousins, hence they also possess twice as many D-branes [1]. For example, since type 0B spectrum has an unrestricted 4-form gauge potential, there are two types of D3-branes: those that couple electrically to this gauge potential, and those that couple magnetically. Very importantly, the weakly coupled spectrum of open strings on type 0 D-branes does not contain tachyons after the GSO projection $(-1)^{F_{o p e n}}=1$ is implemented [1, 11, 33]. Thus, gauge theories living on such D-branes do not have obvious instabilities. This suggests via the 
gauge field/string duality that the bulk tachyon instability of type 0 theory may be cured as well [1, 团.

Let us review an argument for the tachyon stabilization in the simplest setting, which is the stack of $N$ electric and $N$ magnetic D3-branes [⿴囗十. For such a stack the net tachyon tadpole cancels so that there exists a classical solution with $T=0$. In fact, since the stack couples to the selfdual part of the 5-form field strength, the type 0B 3-brane classical solution is identical to the type IIB one. Taking the throat limit suggests that the lowenergy field theory on $N$ electric and $N$ magnetic D3-branes is dual to the $A d S_{5} \times S^{5}$ background of type 0B theory and is therefore conformal in the planar limit [4]. This theory is the $U(N) \times U(N)$ gauge theory coupled to 6 adjoint scalars of the first $U(N), 6$ adjoint scalars of the second $U(N)$, and fermions in the bifundamental representations - 4 Weyl fermions in the $(\mathbf{N}, \overline{\mathbf{N}})$ and 4 Weyl fermions in the $(\overline{\mathbf{N}}, \mathbf{N})$ (the $U(1)$ factors decouple in the infrared). While such a theory does not correspond to placing type IIB D3-branes at an orbifold point, it is nevertheless a $Z_{2}$ projection of the $\mathcal{N}=4 U(2 N)$ gauge theory [4]. The $Z_{2}$ is generated by $(-1)^{F_{s}}$, where $F_{s}$ is the fermion number, together with conjugation by $\left(\begin{array}{cc}I & 0 \\ 0 & -I\end{array}\right)$ where $I$ is the $N \times N$ identity matrix. This is related to the fact that type 0 string theories may be viewed as $(-1)^{F_{s}}$ orbifolds of the corresponding type II theories [5]. In [18] it was pointed out that, since $(-1)^{F_{s}}$ is an element of the center of the $S U(4)$ R-symmetry, this $Z_{2}$ projection of the $\mathcal{N}=4$ theory belongs to the class studied in [25,26].

The fact that the non-supersymmetric AdS/CFT duality considered in 国 is a $Z_{2}$ quotient of the $\mathcal{N}=4$ duality [4, 18] lends additional credence to its validity. In particular, general arguments presented in [34] guarantee that the field theory is conformal in the planar limit and that planar correlators of untwisted vertex operators coincide with those of the $\mathcal{N}=4$ theory. Since this CFT does not appear to have any instabilities at weak coupling, it was argued in [4 that type $0 \mathrm{~B}$ string theory on $A d S_{5} \times S^{5}$ is stable for sufficiently small radius. This provides a simple AdS/CFT argument in favor of tachyon stabilization; it is also an example of how gauge theory may be used to make predictions about the string theory dual to it.

We should note that some important aspects of this particular $Z_{2}$ quotient are related to the twisted sector of the orbifold and are not covered by the analysis in [34]. To analyze some of the physics it will be important that this theory is dual to type $0 \mathrm{~B}$ rather than type IIB background. Therefore, in the usual gravity limit where the radius of $A d S_{5} \times S^{5}$ becomes large in string units and space becomes locally flat, this background is unstable [4]. From the CFT point of view it means that the limit of infinite 't Hooft coupling, which is commonly discussed in the AdS/CFT context, does not make sense. The AdS/CFT correspondence applied to this particular case implies that, as $\lambda=g_{Y M}^{2} N$ is increased, a 
transition should happen at a critical value $\lambda_{c}$ [4]. In this paper we address the origin of this transition from the field theory point of view and argue that it is due to a singularity of a sum over planar diagrams. In non-supersymmetric large $N$ field theories such singularities are expected to occur on general grounds [35. 10 We identify the field theory quantity which we expect to become singular at $\lambda_{c}$ : it is the dimension of the operator which corresponds to the tachyon field via the AdS/CFT map. Using the methods of [6] we show that this operator is

$$
\mathcal{O}_{T}=\frac{1}{4} \operatorname{Tr} F_{\alpha \beta}^{2}-\frac{1}{4} \operatorname{Tr} G_{\alpha \beta}^{2}+\frac{1}{2} \operatorname{Tr}\left(D_{\alpha} X^{i}\right)^{2}-\frac{1}{2} \operatorname{Tr}\left(D_{\alpha} Y^{i}\right)^{2}+\ldots
$$

where $F_{\alpha \beta}$ and $X^{i}$ are the field strength and the 6 adjoint scalars of $S U(N)_{1}$ while $G_{\alpha \beta}$ and $Y^{i}$ are the corresponding objects of $S U(N)_{2}$. Adding such an operator to the action creates a difference between the gauge couplings of $S U(N)_{1}$ and $S U(N)_{2}$. In non-supersymmetric field theory this is not expected to be an exactly marginal deformation. By analyzing 2-loop beta functions we show that the operator (1.1) indeed picks up an anomalous dimension of order $\lambda^{2}$. Higher loops graphs will correct the dimension $\Delta$ as well, and it is conceivable that $\Delta(\lambda)$ develops a singularity at some critical value $\lambda_{c}>0$ as suggested by the AdS considerations.

\section{Anomalous dimension of the tachyon operator}

The standard relation between scalar operator dimension in a conformal field theory and mass in $A d S_{5}$ is $9,10,30$

$$
\Delta=2 \pm \sqrt{4+m^{2} L^{2}}
$$

This relation is definitely valid in the gravity limit where $L^{2} / \alpha^{\prime}=\sqrt{2 \lambda} \gg 1$. Applying this relation to the case of type 0 tachyon, $m^{2}=-2 / \alpha^{\prime}$, we immediately find that for large $\lambda \Delta$ is complex, hence the CFT is unstable. From the AdS point of view, the stability bound [37] that mass-squared must exceed $-4 / L^{2}$ is violated for sufficiently large $L$. For $\lambda$ of order 1 (i.e. for AdS radius of order the string scale) there may be various corrections to the relation (2.1). Even if the overall form of this relation remains the same, $L^{2} / \alpha^{\prime}$ should be treated as some unknown function of $\lambda$, and effective $m^{2}$ might have the form

$$
m^{2}=-\frac{2}{\alpha^{\prime}}+\frac{a_{1}}{L^{2}}+\frac{a_{2} \alpha^{\prime}}{L^{4}}+\ldots
$$

1 In view of this fact, which is also supported by evidence from exactly solvable large $N$ models [36], it may seem surprising that certain non-supersymmetric planar CFT's appear to make sense at infinite 't Hooft coupling [25]. A possible explanation is that in these theories $\lambda_{c}$ is negative. 
This forces us to proceed qualitatively.

To begin with let us simply continue the gravity relation to all $\lambda$ without any change. Then we find

$$
\Delta(\lambda)=2 \pm \sqrt{4-2 \sqrt{2 \lambda}} .
$$

The dimension is real for sufficiently small $\lambda$, and the unitarity bound $\Delta>1$ forces us to pick the positive branch of the square root. This simplified "model" for $\Delta(\lambda)$ implies that $\Delta(0)=4$ which, as we will see, is the correct answer. However, obviously (2.3) cannot be exact: it gives an expansion in powers of $\sqrt{\lambda}$ while in the gauge theory it has to proceed in integer powers of $\lambda$ (we will see that the leading anomalous dimension is $O\left(\lambda^{2}\right)$ ). We expect various corrections to this "model" such as those discussed above. For example, tachyon interactions with the R-R 5-form field strength, which have the form $F_{5}^{2} T^{2}$ [1], give rise to a positive $a_{1}$ in (2.2). One effect of this shift is to push $\lambda_{c}$ to a higher value, but we cannot rule out the presence of other similar corrections. Our qualitative picture is quite insensitive to details however: $\Delta(\lambda)$ is real for $\lambda<\lambda_{c}$ but has a branch cut for $\lambda>\lambda_{c}$ which signals an instability. In the following we will use perturbative field theory arguments to support this scenario.

The primary question is: what is the operator in the CFT on $N$ electric and $N$ magnetic D3-branes that couples to the tachyon. The tachyon coupling to a stack of $N$ electric D3-branes is [1, 3,13 , 13]

$$
-T_{3} \int d^{4} x k_{e}(T)\left(N+\frac{1}{4} \operatorname{Tr} F_{\alpha \beta}^{2}+\frac{1}{2} \operatorname{Tr}\left(D_{\alpha} X^{i}\right)^{2}+\ldots\right),
$$

where $k_{e}(T)=1+\frac{1}{4} T+O\left(T^{2}\right)$ and $T_{3}$ is the tension of an electric D3-brane. The coupling to a stack of $N$ magnetic D3-branes is instead [1, 3,13 ,

$$
-T_{3} \int d^{4} x k_{m}(T)\left(N+\frac{1}{4} \operatorname{Tr} G_{\alpha \beta}^{2}+\frac{1}{2} \operatorname{Tr}\left(D_{\alpha} Y^{i}\right)^{2}+\ldots\right),
$$

where $k_{m}(T)=1-\frac{1}{4} T+O\left(T^{2}\right)$. Adding these two actions, we find that the tachyon coupling to a stack of $N$ electric and $N$ magnetic branes is

$$
\sim \int d^{4} x T\left(\frac{1}{4} \operatorname{Tr} F_{\alpha \beta}^{2}-\frac{1}{4} \operatorname{Tr} G_{\alpha \beta}^{2}+\frac{1}{2} \operatorname{Tr}\left(D_{\alpha} X^{i}\right)^{2}-\frac{1}{2} \operatorname{Tr}\left(D_{\alpha} Y^{i}\right)^{2}+\ldots\right)
$$

leading to the expression (1.1) for the gauge invariant operator corresponding to the tachyon field via the AdS/CFT map. This operator is odd under the interchange of the two $S U(N)$ 's which is related to the fact that the tachyon is one of the twisted states from the point of view of orbifolding type IIB string theory by $(-1)^{F_{s}}$. Indeed, the tachyon belongs to the $(N S-, N S-)$ sector of type 0B theory which is not present in the type 
IIB spectrum. In other $Z_{2}$ orbifold theories it was also found that twisted states couple to vertex operators odd under the interchange of the factors of the gauge group [38,28]. Fields from the untwisted sector, on the other hand, couple to operators that are even under the interchange of the gauge groups: for example, the dilaton couples to

$$
\frac{1}{4} \operatorname{Tr} F_{\alpha \beta}^{2}+\frac{1}{4} \operatorname{Tr} G_{\alpha \beta}^{2}+\ldots .
$$

In order to study the anomalous dimension of the operator (1.1) at weak coupling, we may consider slightly unequal gauge couplings for $S U(N)_{1}$ and $S U(N)_{2}$. Then the coupling constant for this operator is

$$
T=\frac{1}{\lambda_{1}}-\frac{1}{\lambda_{2}},
$$

where $\lambda_{i}=g_{i}^{2} N$, and $i=1,2$. The planar (leading order in $N$ ) renormalization group equations to two-loop order are [4]

$$
\begin{aligned}
& \mu \frac{\partial}{\partial \mu} \frac{1}{\lambda_{1}}=a\left(\lambda_{1}-\lambda_{2}\right)+O\left[\left(\lambda_{1}-\lambda_{2}\right)^{2}\right], \\
& \mu \frac{\partial}{\partial \mu} \frac{1}{\lambda_{2}}=a\left(\lambda_{2}-\lambda_{1}\right)+O\left[\left(\lambda_{1}-\lambda_{2}\right)^{2}\right],
\end{aligned}
$$

and we find $a=-(2 \pi)^{-4}$. Due to the vanishing of the one-loop beta function there is no constant term on the right-hand side of the equations. The two-loop contributions vanish for equal couplings, as shown explicitly in [4], but they no longer vanish for $\lambda_{1} \neq \lambda_{2}$.

Taking the difference of the two equations, and working to first order in $T$, we have

$$
\mu \frac{\partial T}{\partial \mu}=\frac{\lambda^{2}}{8 \pi^{4}} T .
$$

Solving this we have

$$
T=T_{0}\left(\frac{\mu}{\Lambda}\right)^{\frac{\lambda^{2}}{8 \pi^{4}}} .
$$

Since $T$ grows in the UV (for $\mu \gg \Lambda$ ) the associated operator (1.1) is irrelevant to this order: its dimension is

$$
\Delta(\lambda)=4+\frac{\lambda^{2}}{8 \pi^{4}}+O\left(\lambda^{3}\right) .
$$

Based on the AdS/CFT correspondence, we conjecture that further corrections to $\Delta(\lambda)$ will produce a function which develops a singularity at $\lambda_{c}>0$. We do not expect direct calculations of the anomalous dimension to be feasible beyond $O\left(\lambda^{3}\right)$ which may be extracted from 3-loop beta functions. However, it might be possible to put bounds on the general $O\left(\lambda^{n}\right)$ terms and prove the existence of a critical point at $\lambda_{c}>0$. 
To summarize this paper, we have suggested that finite radius of convergence of planar graphs, which is a well-known phenomenon in large $N$ field theories, has interesting implications in view of the AdS/CFT correspondence. Namely, if there is an operator whose dimension is real up to a critical value of the 't Hooft coupling but becomes complex beyond that value, then the dual AdS phenomenon is that a tachyon present in the large radius (gravity) limit is stabilized for sufficiently small radii. It is difficult to study such backgrounds with string-scale curvature directly, but on the dual CFT side perturbative calculations are quite tractable. The perturbative stability of the CFT thus provides an argument for stabilization of tachyons (this is one of the few results to date where field theory has been used to make new predictions about string theory), and finiteness of the radius of convergence of planar graphs suggests how instability may develop at sufficiently strong coupling. We have discussed one specific example which arises in the context of type 0 theory but we hope that these phenomena are quite general.

\section{Acknowledgements}

I am grateful to E. Silverstein, A. Tseytlin and especially J. Minahan for useful discussions and comments. This work was supported in part by the NSF grant PHY-9802484 and by the James S. McDonnell Foundation Grant No. 91-48. 


\section{References}

[1] I.R. Klebanov and A.A. Tseytlin, "D-Branes and Dual Gauge Theories in Type 0 Strings," Nucl. Phys. B546 (1999) 155, hep-th/9811035.

[2] J. Minahan, "Glueball Mass Spectra and Other Issues for Supergravity Duals of QCD Models," hep-th/9811156.

[3] I.R. Klebanov and A.A. Tseytlin, "Asymptotic Freedom and Infrared Behavior in the Type 0 String Approach to Gauge Theory," Nucl. Phys. B547 (1999) 143, hepth/9812089.

[4] I.R. Klebanov and A.A. Tseytlin, "Non-supersymmemtric CFT from Type 0 String Theory," J. High Energy Phys. 9903 (1999) 015, hep-th/9901101.

[5] L. Dixon and J. Harvey, "String theories in ten dimensions without space-time supersymmetry", Nucl. Phys. B274 (1986) 93; N. Seiberg and E. Witten, "Spin structures in string theory", Nucl. Phys. B276 (1986) 272; C. Thorn, unpublished.

[6] I.R. Klebanov, "World volume approach to absorption by nondilatonic branes," Nucl. Phys. B496 (1997) 231, hep-th/9702076; S.S. Gubser, I.R. Klebanov, and A.A. Tseytlin, "String theory and classical absorption by three-branes," Nucl. Phys. B499 (1997) 217, hep-th/9703040.

[7] S.S. Gubser and I.R. Klebanov, "Absorption by branes and Schwinger terms in the world volume theory," Phys. Lett. B413 (1997) 41, hep-th/9708005.

[8] J. Maldacena, "The Large N limit of superconformal field theories and supergravity," Adv. Theor. Math. Phys. 2 (1998) 231, hep-th/9711200.

[9] S.S. Gubser, I.R. Klebanov, and A.M. Polyakov, "Gauge theory correlators from noncritical string theory," Phys. Lett. B428 (1998) 105, hep-th/9802109.

[10] E. Witten, "Anti-de Sitter space and holography," Adv. Theor. Math. Phys. 2 (1998) 253 , hep-th/9802150.

[11] A.M. Polyakov, "The Wall of the Cave," hep-th/9809057.

[12] A.M. Polyakov, "String theory and quark confinement," Nucl. Phys. B (Proc. Suppl.) 68 (1998) 1 , hep-th/9711002.

[13] M.R. Garousi, "String Scattering from D-branes in Type 0 Theories", hep-th/9901085.

[14] M. Billó, B. Craps and F. Roose, "On D-branes in Type 0 String Theory," hepth/9902196.

[15] K. Zarembo, "Coleman-Weinberg Mechanism and Interaction of D3-branes in Type 0 String Theory, hep-th/9901106; A. Tseytlin and K. Zarembo, "Effective Potential in Non-supersymmetric $S U(N) \times S U(N)$ Gague Theory and Interactions of Type 0 D3-branes," hep-th/9902095.

[16] G. Ferretti and D. Martelli, "On the construction of gauge theories from non critical type 0 strings," hep-th/9811208. 
[17] J. Minahan, "Asymptotic Freedom and Confinement from Type 0 String Theory," hep-th/9902074.

[18] N. Nekrasov and S. Shatashvili, "On non-supersymmetric CFT in four dimension," hep-th/9902110.

[19] M. Costa, "Intersecting D-branes and black holes in type 0 string theory," hepth/9903128.

[20] M. Alishahiha, A. Brandhuber and Y. Oz, "Branes at Singularities in Type 0 String Theory," hep-th/9903186.

[21] O. Bergman and M. Gaberdiel, "Dualities of Type 0 Strings," hep-th/9906055.

[22] A. Armoni and B. Kol, "Non-supersymmetric Large $N$ Gauge Theories from Type 0 Brane Configurations," hep-th/9906081.

[23] R. Blumenhagen, A. Font and D. Lust, "Non-Supersymmetric Gauge Theories from D-Branes in Type 0 String Theory," hep-th/9906101.

[24] M. Douglas and G. Moore, "D-branes, quivers, and ALE instantons," hep-th/9603167.

[25] S. Kachru and E. Silverstein, "4d conformal field theories and strings on orbifolds," Phys. Rev. Lett. 80 (1998) 4855, hep-th/9802183.

[26] A. Lawrence, N. Nekrasov and C. Vafa, "On conformal field theories in four dimensions," Nucl. Phys. B533 (1998) 199, hep-th/9803015.

[27] A. Kehagias, "New Type IIB Vacua and Their F-Theory Interpretation," hepth/9805131.

[28] I.R. Klebanov and E. Witten, "Superconformal field theory on threebranes at a CalabiYau singularity," Nucl. Phys. B536 (1998) 199, hep-th/9807080; S.S. Gubser, "Einstein manifolds and conformal field theories," hep-th/9807164.

[29] D.R. Morrison and M.R. Plesser, "Non-Spherical Horizons, I," hep-th/9810201.

[30] I.R. Klebanov and E. Witten, "AdS/CFT Correspondence and Symmetry Breaking," hep-th/9905104.

[31] P. Frampton, "ADS/CFT String Duality and Conformal Gauge Theories," hepth/9812117; P. Frampton and C. Vafa, "Conformal Approach to Particle Phenomenology," hep-th/9903226.

[32] C. Csaki, W. Skiba and J. Terning, "Beta Functions of Orbifold Theories and the Hierarchy Problem," hep-th/9906057.

[33] O. Bergman and M. Gaberdiel, "A Non-supersymmetric Open String Theory and S-Duality," Nucl. Phys. B499 (1997) 183, hep-th/9701137.

[34] M. Bershadsky, Z. Kakushadze and C. Vafa, "String expansion as large N expansion of gauge theories", Nucl. Phys. B523 (1998) 59, hep-th/9803076; M. Bershadsky and A. Johansen, "Large N limit of orbifold field theories," Nucl. Phys. B536 (1998) 141, hep-th/9803249.

[35] G. "t Hooft, "On the Convergence of Planar Diagram Expansions," Comm. Math. Phys. 86 (1982) 449. 
[36] E. Brezin, C. Itzykson, G. Parisi and J. Zuber, Comm. Math. Phys. 59 (1978) 35.

[37] P. Breitenlohner and D.Z. Freedman, "Stability in gauged extended supergravity", Ann. Phys. 144 (1982) 249.

[38] S. Gukov, "Comments on N=2 AdS Orbifolds," Phys. Lett. B439 (1998) 23, hepth/9906081. 